\title{
Preface: emergent coatings and thin films for high-temperature energy applications
}

\author{
Jose Luis Endrino ${ }^{1,2}$ \\ Published online: 8 December 2021 \\ (c) Qatar University and Springer Nature Switzerland AG 2021
}

Increasing the temperature capability of mechanical devices and components is a major requirement of the energy sector. The development of new coating and thin film compositions is of strategic importance for a wide range of energy applications, which can directly influence the transport, aerospace, and manufacturing industries. This special issue covers the design and development of innovative materials related to high-temperature energy conversion, conservation, harvesting, and storage.

Among the most important challenges to be solved before the implementation of a high-temperature energy conversion, conservation, harvesting, and storage technologies as feasible alternatives in the market is the development of new material solutions which can withstand the harsh environmental requirements and deliver proper functionality. Clearly, the variability of the heat source which sometimes comes from the fluctuation of the renewable source makes the materials challenges of critical importance. The large variation in operational temperature and the harsh environmental requirement of each application means that the match of thermal properties between substrate and coating materials needs to be optimal. Thus, emergent materials and coatings need to be designed to withstand aggressive environments due to new requirements of large temperature gradients, cyclic stress, and corrosive atmospheres.

Proper multifunctional performance while maintaining an acceptable cost is an implicit requirement among hightemperature energy applications. In this manner, low-cost solutions involving coating materials that can be scaled up and deployed at a large scale are needed in the field of solar selective coatings, energy conversion, and storage.

Jose Luis Endrino

jose.endrino@nano4energy.eu

1 Nano4Energy SL, José Gutiérrez Abascal 2, 28006 Madrid, Spain

2 Universidad Loyola Andalucia, Av de las Universidades s/n, 41704 Dos Hermanas, Sevilla, Spain
Therefore, a lot of efforts are being put on trying to manufacture new coating materials using less expensive production methods such as thermal spraying.

Due to the critical importance of materials in the performance of high-temperature energy systems, we recently launched the present special issue (SI) entitled "Emergent Coatings and Thin Films for High Temperature Energy Applications" which underscores some of the newest research and development at the forefront of this field. Authors from around the globe were invited to submit contributions addressing some of these important matters. Although there has recently been a significant number of contributions, both research articles and reviews, that cover single topics related to high-temperature energy applications, such as high-temperature energy storage, this SI focuses on emergent coatings related to the general area of high-temperature energy applications from a materials science and engineering standpoint. The four "hot" topics covered in this SI of Emergent Materials are (i) the role of thermal barrier and optical coatings deposited with spray and physical vapor deposition techniques for combating climate change and improving the performance as thermal barriers or for corrosion protection; this first topic is also the main topic of the SI, (ii) recent developments in metallic materials for joining or filtering that can meet the new demands of various high-temperature energy applications, (iii) the role of nanomaterials and surface functionalization techniques on the synthesis of novel nanofluids with improved heat transfer properties, and (iv) the role of metrology and machine learning in the manufacturing of new coatings and nanomaterials.

Two invited review articles, covering the most recent stateof-the-art, are presented in this special issue. The reviews highlight the importance of advanced surface metrology tools in studying the nanomechanical properties of coatings near their temperature of operation and the importance of thermal spray deposition techniques in combating climate change. These two review articles are timely and have a vital importance in the field of materials development for hightemperature energy applications.

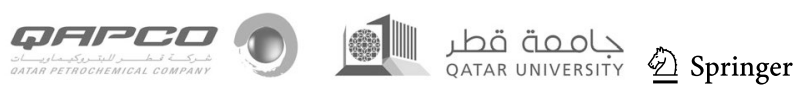

